ISSN 1409-2441
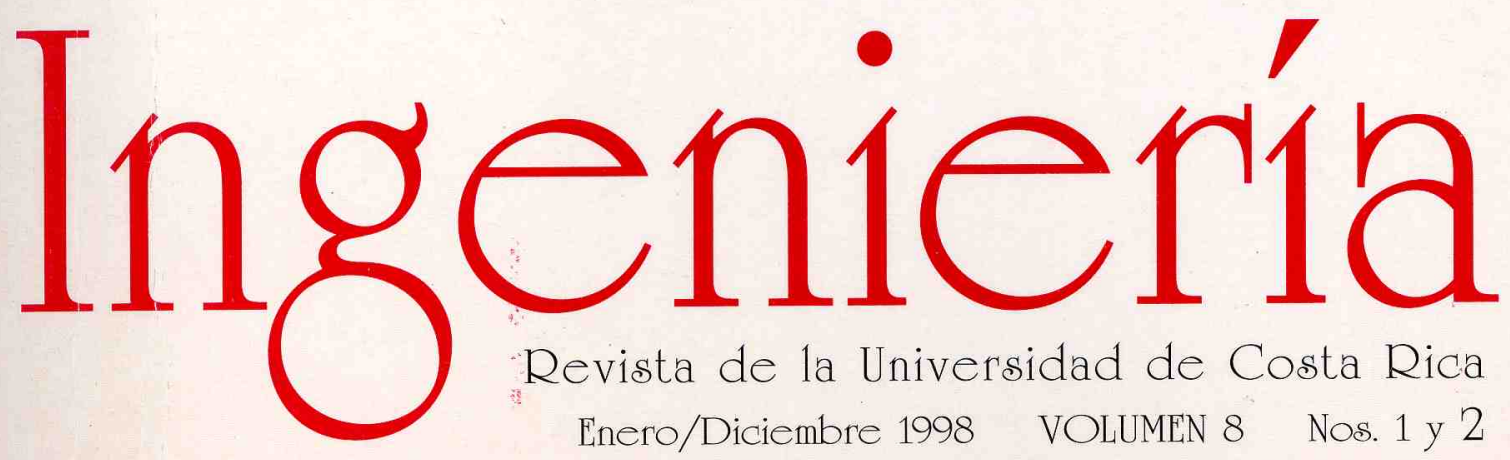

Revista de la Universidad de Costa Rica Enero/Diciembre 1998 VOLUMEN 8 Nos. 1 y 2 


\title{
CONTROL DE CALIDAD DEL CONCRETO ESTRUCTURAL Y DEL MORTERO DE PEGA EN VIVIENDAS
}

\author{
Mauricio Araya $R$. \\ Flor de María Muñoz U.1
}

\begin{abstract}
Resumen
Ennuestropaís. las mezclas de concreto estmctural y mortero de pega más utilizadas en viviendas de uno y dos pisos son las que se elaboranen el sitio de constmcción. La resistencia [mal en ambos casos, depende de factores que intervienen tanto en la tase de produccióncomo en la de colocación. Entre estos tenemos principalmente: la mano de obra, el equipo disponible, la calidad de los materialesy la supervisión profesional. En este trabajo se identitica la mala práctica que se presenta durante la preparación de uno u otro tipo de mezcla y que incide negativamente en la calidad de las mismas. Además se incorpora información técnica que puede servircomo guía práctica a la hora de dositicar o variar las proporciones de los materiales en el sitio. Los resultados compmeban que en estetipo de obras se presentan deficiencias que afectan la resistencia [mal del concreto y que reducen la probabilidad de obtener concretosy morteros que cumplan con las especificaciones que exige el Código Sísmico de Costa Rica.
\end{abstract}

\section{Summar)'}

In our l'Ountry, the stmctural concrete mixes and binding mortar more comrnonly used in houses of one or two t100rs are the ones madein the place of constmetion. The [mal resistance in both cases, depend5 on factors that occur either in the phase of production or in tbe puning in place. Among these we have: workmanship, equipment avaliable, quality of materials, and professional supervision.This study identifies the mistakes or carelessnesses that most frequently occur during the preparation of one or the other type01'mixes and that have a negative effect on their quality. It also provides technical information that can be useful as a practical guidewhen proportioning or when making variations in the proportions of materials in the place of constmction. TIIe results confiml lhat in this type of works there are deticiencies that affect the tmal resistance of mixes and decrease the probability of obtaining concrdeand mortar that meet the specifications required in the Sismic Code of Costa Rica.

\section{INTRODUCCIÓN}

Losconcretosestructurales y morteros de pega quese realizan en el sitio, no llegan a cumplir con la resistencia que se especifica en las normas de construcción o en los planos constructivos, lo que produce deficiencias potencialesen elementos estructurales tales comovigas, columnas, entrepisos y muros, y que pueden llegar a constituir un grave problemale seguridad.
La resistencia del concreto confeccionado en obra está gobernada por las condiciones que se dan desde el diseño y la selección de los materiales, hasta su fabricación y colocación. En este sentido es de suma importancia para la persona responsable del proyecto, contar con la infonnación básica necesaria para diseñar la mezcla y hacer las recomendaciones pertinentes durante su preparación y colocación.

La falta de mano de obra calificada en el sector construcción en Costa Rica es un

1 Investigadores del Instituto de Investigaciones en Ingeniería, U.C.R. 
problema constante. que se agudiza en épocas de alta demanda constructiva. Este trabajo es un documento base que señala los errores y malos hábitos detectados en sitio, a partir de una muestra representativa del tipo de edificación analizada durante la preparación y colocación del concreto y el mortero.

\section{DEFINICIÓN Y UBICACIÓN DE LA MUESTRA}

Esta investigación comprende un muestreo en obra del concreto estructural. mortero de pega, agregados y el cemento. utilizados en su elaboración. correspondientes a construcciones de viviendas de uno y dos pisos tomadas al azar dentro del Área Metropolitana de San José. La distribución de espeCÍmenes fue la siguiente:

$\begin{array}{lll}\text { Sector Norte: } & \text { Tibás } & \text { (3 espeCÍmenes) } \\ & \text { Moravia } & \text { (3 espeCÍmenes) } \\ \text { Sector Sur: } & \begin{array}{l}\text { Desamparados } \\ \text { (3 espeCÍmenes) }\end{array} \\ \text { San Francisco } & \text { (3 espeCÍmenes) } \\ \text { Sector Oeste: } & \text { Escazú } & \begin{array}{l}(3 \text { espeCÍmenes) } \\ \text { (3 espeCÍmenes) } \\ \text { Pavas }\end{array} \\ \text { Sector Este: } & \text { Curridabat } & \text { (3 especímenes) } \\ & \text { Tres Ríos } & \text { (3 espeCÍmenes) } \\ \text { Sector Central:Zapote } & \text { (3 especímenes) } \\ & \text { San Pedro } & \text { (3 espeCÍmenes) }\end{array}$

En los espeCímenes de concreto y mortero el parámetro de comparación principal fue la resistencia a la compresión uniaxial. que se especifica a 28 dias ( $\left.f^{\prime} c\right)$. El tipo de construcción considerada en el estudio fue únicamente de carácter privado; se excluyen las viviendas de interés social.

\section{PRUEBAS DE LABORATORIO}

Los especímenes para prueba proceden de las mezclas de concreto y mortero producidas en cada obra, para reflejar, de la manera más real posible, las condiciones presentes en el campo.

\subsection{Concreto estructural:}

Los testigos (cilindros de $150 \mathrm{~mm} \times 300 \mathrm{~mm}$ ) se curaron en cámara húmeda y se fallaron a 28 dias; el valor de resistencia obtenido es el promedio de los tres valores. El procedimiento que se siguió para valorar la calidad del concreto fue el siguiente:

1. Recolección de la infonnación referente a la mezcla de la que se extrajo cada espécimen de concreto y se le asignó un código de identificación a cada uno.

2. Ejecución de la prueba AS.T.M. C 14390: "Prueba de revenimiento del concreto con el cono de Abrahams".

3. Aplicación de la nonna AS.T.M. C 31-91: "Práctica estándar para preparar y curar espeCÍmenes de concreto en sitio".

4. Se prepararon los cilindros según la especificación AS.T.M. C 617-94: "Cabeceo de espeCÍmenes cilíndricos de concreto".

5. Finalmente se pesaron las muestras para luego fallarlas siguiendo el procedimiento descrito por la nonna AS.T.M. C 39-86: "Resistencia a la compresión uniaxial de espeCÍmenes cilíndricos de concreto".

\subsection{Mortero de pega:}

El procedimiento seguido comprendió:

1. Recolección de la infonnación referente a la mezcla de la que se extrajo cada espécimen de mortero y se le asignó un código de identificación a cada uno.

2. Confección de los cubos de prueba con base en la norma AS.T.M. C 109-93: "Método estándar de prueba de compresión uniaxial para morteros de cemento hidráulico" .

3. Al cumplir veintiocho dias de curado en la cámara húmeda, se procedió a fallar los cubos en la máquina Universal. 
3.3. Agregado grueso:

Serealizaron las cuatro pruebas siguientes:

- AS.T.M. C 127: "Pesos específicos y absorción de agregados gruesos".

- AS.T.M. C 29: "Pesos unitarios".

- AS.T.M. C 136: "Análisis granulométrico" .

- A.S.T.M. C 131: "Prueba de abrasión en máquina Los Ángeles".

\subsection{Agregado fino:}

También con base en las pruebas que se indican,se procedió a su caracterización:

- AS.T.M. C 128: "Pesos espeCÍficos y absorción de agregados finos".

- AS.T.M. C 29: "Pesos unitarios".

- A.S.T.M. C 136: "Análisis granulométrico" .

- AS.T.M. C 40: "Prueba de colorimetría".

\subsection{Cemento tipo $1 \mathrm{MP}$ :}

Todaslas construcciones involucradas en esta investigación utilizaron cemento tipo $1 \mathrm{MP}$ (cementportland modificado con puzolanas), por ser el único disponible comercialmente en elpaís. Las pruebas a que se sometió fueron:
- AS.T.M. C 187: "Prueba de consistencia normal de un cemento hidráulico".

- AS.T.M. C 191: "Prueba de tiempo de fraguado de un cemento hidráulico".

- AS.T.M. C 188: "Peso específico de un cemento hidráulico".

En nuestro país el cemento portland tipo $1 \mathrm{MP}$ se puede adquirir en sacos de papel kraft con un peso de $50 \mathrm{~kg}$ Y su contenido prácticamente llena un cubo de $37 \mathrm{~cm}$ de lado, lo que implica un incremento de volumen con respecto al cemento portland tipo 1 , esto obedece a la reducción del peso unitario masivo seco de 1 $500 \mathrm{kglm}^{3}$ en el tipo 1 hasta $980 \mathrm{~kg} / \mathrm{m}^{3}$ para el tipo $1 \mathrm{MP}$. Un saco de cemento portland tipo 1 de $50 \mathrm{~kg}$ llena un cajón de $33 \mathrm{~cm}$ de lado.

\section{RESISTENCIAS DEL CONCRETO y EL MORTERO}

El promedio de las resistencias a la compresión uniaxial para cada una de las obras se presenta en el cuadro $\mathrm{N}^{\circ} 1$. A partir de esos datos se clasifican las mezclas en función de sus cualidades como se muestra en los cuadros 2 y 3 .

Cuadro $\mathrm{N}^{\circ} 1$. Resistencias promedio a la compresión para el concreto y el mortero muestreado en 30 viviendas del Área Metropolitana de San José.

\begin{tabular}{|c|c|c|}
\hline \multirow{2}{*}{$\begin{array}{c}\text { Construcción } \\
\#\end{array}$} & $\begin{array}{c}\text { Cilindros de concreto } \\
\text { Resistencia promedio } \\
\text { a la compresión } \\
\text { a 28 días }\left(\mathrm{kg} / \mathrm{cm}^{2}\right)\end{array}$ & $\begin{array}{c}\text { Cubos de mortero } \\
\text { a la compresión } \\
\text { a } 28 \text { dias }\left(\mathrm{kg} / \mathrm{cm}^{2}\right)\end{array}$ \\
\hline 1 & 110 & 148 \\
\hline 2 & 164 & 216 \\
\hline 3 & 186 & 125 \\
\hline 4 & 78 & 221 \\
\hline 5 & 272 & 162 \\
\hline 6 & 155 & 163 \\
\hline 7 & 141 & 242 \\
\hline
\end{tabular}




\begin{tabular}{|c|c|c|}
\hline 8 & 67 & 228 \\
\hline 9 & 160 & 268 \\
\hline 10 & 102 & 189 \\
\hline 11 & 107 & 274 \\
\hline 12 & 127 & 221 \\
\hline 13 & 87 & 114 \\
\hline 14 & 210 & 139 \\
\hline 15 & 252 & 164 \\
\hline 16 & 265 & 306 \\
\hline 17 & 106 & 130 \\
\hline 18 & 96 & 171 \\
\hline 19 & 178 & 216 \\
\hline 20 & 171 & 188 \\
\hline 21 & 138 & 151 \\
\hline 22 & 86 & 189 \\
\hline 23 & 217 & 116 \\
\hline 24 & 141 & 219 \\
\hline 25 & 86 & 145 \\
\hline 26 & 161 & 156 \\
\hline 27 & 111 & 146 \\
\hline 28 & $J 17$ & 203 \\
\hline 49 & 121 & 210 \\
\hline 30 & 153 & 153 \\
\hline & & \\
\hline & & \\
\hline
\end{tabular}

Cuadro $\mathrm{N}^{\circ} 2$. Clasificación del concreto estructural en función de su resistencia a la compresión a 28 dias.

\begin{tabular}{|c|c|c|c|c|}
\hline Calidad & $\begin{array}{c}\text { Resistencia a la } \\
\text { compresión }\left(\mathrm{k} 2 \mathrm{fcm}^{2}\right)\end{array}$ & $\begin{array}{c}\text { Cantidad de } \\
\text { obras }\end{array}$ & $\begin{array}{c}\% \text { de } \\
\text { obras }\end{array}$ & Conclusión \\
\hline Muy mala & $0-100$ & 6 & 20 & no cumple \\
\hline Mala & $100-175$ & 17 & 56 & no cumple \\
\hline $\begin{array}{c}\text { Regular, podrla cumplir } \\
\text { a un plazo mayor }\end{array}$ & 175 a menos de 210 & 2 & 7 & no cumple \\
\hline Buena & más de 210 & 5 & 17 & sí cumple \\
\hline
\end{tabular}

Cuadro $\mathrm{N}^{\circ} 3$. Clasificación del mortero de pega en función de su resistencia a la compresión a 28 dias (AS.T.M. C 270).

\begin{tabular}{|c|c|c|c|c|}
\hline Calidad & $\begin{array}{c}\text { Resistencia a la } \\
\text { compresión } \\
\left(\mathrm{k} 2 / \mathrm{cm}^{2}\right)\end{array}$ & $\begin{array}{l}\text { Cantidad } \\
\text { de obras }\end{array}$ & $\begin{array}{l}\% \text { de } \\
\text { obras }\end{array}$ & Conclusión \\
\hline Muy mala (tipo O) & $25-53$ & 0 & 0 & no cumple \\
\hline Mala (tipo $\mathrm{N}$ ) & $53-126$ & 3 & 10 & no cumple \\
\hline
\end{tabular}




\begin{tabular}{|c|c|c|c|c|}
\hline $\begin{array}{c}\text { Buenqara usar en } \\
\text { mamposteríatipo S) }\end{array}$ & $\begin{array}{c}126 \text { a menos de } \\
175\end{array}$ & 12 & 40 & sí cumple \\
\hline $\begin{array}{c}\text { Buenpara usar en } \\
\text { mampostería(tipo } \\
\text { M) }\end{array}$ & más de 175 & 15 & 50 & sí cumple \\
\hline
\end{tabular}

Delos cuadros $\mathrm{N}^{\circ} 2$ y $\mathrm{N}^{\circ} 3$ se evidencia el bajo porcentaje de obras que cumplen con la resistencia estipuladæn el Código Sísmico de Costa Rica. Para el caso del concreto, solamente el $17 \%$ lo cumplió.En el mortero, el porcentaje de cumplimiento es mayor $(50 \%)$ si se toma como parámetro decomparaciónel antiguo mortero tipo PL, para el cual se especificaba una resistencia de $175 \mathrm{~kg} / \mathrm{cm}^{2}$ a 28 días. No obstante, el porce taje de morteros que cumplen con la resistencia mínima aceptable parasu uso en mampostería (tipo M Y S) es del 90\%.

Enlos cuadros $\mathrm{N}^{\circ} 4$ y $\mathrm{N}^{\circ} \mathrm{S}$ aparecen, para cada obra, la dosificación volumétrica utilizada para el concrety mortero respectivamente.

Cuadro $\mathrm{N}^{\circ} 4$. Resumen de los factores más importantes relacionados con la preparación del concreto en las 30 obras analizadas. Área Metropolitana de San José.

\begin{tabular}{|c|c|c|c|c|c|}
\hline $\begin{array}{c}\text { Obra } \\
\#\end{array}$ & Lugar & $\begin{array}{c}\mathrm{f}^{\prime} \mathrm{e} \\
\left(\mathrm{k} 2 / \mathrm{em}^{z}\right)\end{array}$ & $\begin{array}{c}\text { Dosif. Vol. } \\
(\mathrm{C}: \mathrm{A}: \mathrm{P})\end{array}$ & $\begin{array}{c}\text { Revenimiento } \\
(\mathrm{cm})\end{array}$ & Equipo \\
\hline 1 & Tres Ríos & $11 \mathrm{O}$ & $1: 2: 4$ & $18, \mathrm{O}$ & batidora \\
\hline 2 & Tres Ríos & 164 & $1: 2: 4$ & 21.0 & batidora \\
\hline 3 & Tres Rios & 186 & $1: 3: 2$ & 18,0 & batidora \\
\hline 4 & Curridabat & 78 & $1: 3: 2$ & 21.5 & batidora \\
\hline 5 & Curridabat & 272 & $1: \mathrm{U}: 3$ & 14.0 & batidora \\
\hline 6 & Curridabat & 155 & $1: 2,5: 3$ & $21, \mathrm{O}$ & batidora \\
\hline 7 & San Francisco & 141 & $1: 2: 4$ & 40 & batidora' \\
\hline 8 & San Francisco & 67 & $1: 3: 3$ & 210 & manual \\
\hline 9 & San Francisco & 160 & $1: 2: 3$ & 40 & manual \\
\hline 10 & Desamnarados & 102 & $1: 2: 2$ & 210 & batidora \\
\hline 11 & Desamnarados & 107 & $1: 2: 3$ & $18, \mathrm{O}$ & batidora \\
\hline 12 & Desamparados & 127 & $1: 2: 3$ & 20.0 & manual \\
\hline 13 & Zanote & 87 & $1: 3: 4$ & $20, \mathrm{O}$ & batidora \\
\hline 14 & Zanote & 210 & $1: 2: 3$ & 210 & batidora \\
\hline 15 & Zaoote & 252 & $1: 2: 3$ & 70 & batidora \\
\hline 16 & San Pedro & 265 & $1: 2: 4$ & $15, \mathrm{O}$ & batidora \\
\hline 17 & San Pedro & 106 & $1: 3: 3$ & $16, \mathrm{O}$ & batidora \\
\hline 18 & San Pedro & 96 & $1: 4: 3$ & 225 & batidora \\
\hline 19 & Tibás & 178 & $1: 2: 3$ & $19, \mathrm{O}$ & batidora \\
\hline 20 & Tibás & 171 & $1: 2: 4$ & $20, \mathrm{O}$ & manual \\
\hline 21 & Tibás & 138 & $1: 3: 3$ & 5,5 & manual \\
\hline 22 & Moravia & 86 & $1: 3: 3$ & $20, \mathrm{O}$ & batidora \\
\hline
\end{tabular}




\begin{tabular}{|c|c|c|c|c|c|}
\hline 23 & Moravia & 217 & $1: 2: 3,5$ & 15,0 & batidora \\
\hline 24 & Moravia & 141 & $1: 2,5: 2,5$ & 125 & illanual \\
\hline 25 & Escazú & 86 & $1: 2: 2$ & 18,0 & manual \\
\hline 26 & Escazú & 161 & $1: 2: 3$ & 18,0 & manual \\
\hline 27 & Escazú & 111 & $1: 2: 2$ & 18,0 & batidora \\
\hline 28 & Pavas & 117 & $1: 2: 3$ & 18,0 & batidora \\
\hline 29 & Pavas & 121 & $1: 2: 4$ & 18,0 & manual \\
\hline 30 & Pavas & 153 & $1: 2: 3$ & 8,0 & manual \\
\hline
\end{tabular}

Cuadro $\mathrm{N}^{\circ} 5$. Resumen de los factores más importantes relacionados con la preparación del mortero en las 30 obras analizadas. Área Metropolitana de San José.

\begin{tabular}{|c|c|c|c|c|}
\hline $\begin{array}{c}\text { Obra } \\
\# \\
\end{array}$ & Lugar & $\begin{array}{l}\text { Resist. } 28 \text { días } \\
\left(\mathrm{k} 2 / \mathrm{cm}^{2}\right)\end{array}$ & $\begin{array}{l}\text { Dosif. Vol. } \\
\text { (C: A) }\end{array}$ & Equipo \\
\hline 1 & Tres Ríos & 148 & $1: 4$ & manual \\
\hline 2 & Tres Rios & 216 & $1: 2,5$ & batidora \\
\hline 3 & Tres Ríos & 125 & $1: 3$ & batidora \\
\hline 4 & Curridabat & 221 & $1: 2,5$ & batidora \\
\hline 5 & Curridabat & 162 & $1: 3$ & manual \\
\hline 6 & Curridabat & 163 & $1: 3,5$ & batidora \\
\hline 7 & San Francisco & 242 & $1: 3$ & manual \\
\hline 8 & San Francisco & 228 & $1: 3$ & manual \\
\hline 9 & San Francisco & 268 & $1: 3$ & manual \\
\hline 10 & Desamparados & 189 & $1: 3$ & batidora \\
\hline 11 & Desamparados & 274 & $1: 3$ & batidora \\
\hline 12 & Desamparados & 221 & $1: 3$ & manual \\
\hline 13 & Zaoote & 114 & $1: 3,5$ & manual \\
\hline 14 & Zaoote & 139 & $1: 4$ & batidora \\
\hline 15 & Zaoote & 164 & $1: 3$ & batidora \\
\hline 16 & San Pedro & 306 & $1: 2$ & manual \\
\hline 17 & San Pedro & 130 & $1: 3$ & batidora \\
\hline 18 & San Pedro & 171 & $1: 3$ & batidora \\
\hline 19 & Tibás & 216 & $1: 3$ & batidora \\
\hline 20 & Tibás & 188 & $1: 3$ & manual \\
\hline 21 & Tibás & 151 & $1: 3.5$ & manual \\
\hline 22 & Moravia & 189 & $1: 3$ & batidora \\
\hline 23 & Moravia & 116 & $1: 4$ & batidora \\
\hline 24 & Moravia & 219 & $1: 2,5$ & manual \\
\hline 25 & Escazú & 145 & $1: 3$ & manual \\
\hline 26 & Escazú & 156 & $1: 4$ & manual \\
\hline 27 & Escazú & 146 & $1: 4$ & manual \\
\hline 28 & Pavas & 203 & $1: 3$ & manual \\
\hline 29 & Pavas & 210 & $1: 2,5$ & manual \\
\hline 30 & Pavas & 153 & $1: 3,5$ & manual \\
\hline
\end{tabular}


EliJasdosificaciones volumétricas de los cuadros $\mathrm{N}^{\circ} 4$ y $\mathrm{N}^{\circ} \mathrm{S}$ el número correspondiente al cemento portland tipo $1 \mathrm{MP}$ se refiere a sacos completos de $50 \mathrm{~kg}$ Y no a cajones de $33 \mathrm{~cm}$ de lado, los que sí fuerontilizados para medir los agregados.

Delanálisis de la infonnación del cuadro $\mathrm{N}^{\circ} 4$ se tiene que:

- Ninguna de las mezclas de concreto preparadas a mano cumplen con la resistencia que se especificaen planos constructivos para 28 dias $\left(210 \mathrm{kglcm}^{2}\right)$

- Ladosificación más utilizada es por volumen de $1: 2: 3$ (cemento: arena: piedra con el cemento dosificadopor saco completo de $50 \mathrm{~kg}$ y los agregados en cajones de $33 \mathrm{~cm}$ de lado), la cual estuvo presente en el $30 \%$ de los casos. A la vez, el revenimiento que más veces se repite es de $18,0 \mathrm{~cm}$ y seda en el $27 \%$ de las obras .

- A pesar de que en el $67 \%$ de las obras se cuenta con una batidora para preparar la mezcla de concreto. la cantidad de agua para hacer la revoltura trabajable fue excesiva, lo que generó revenimientos muy altos en general. En el cuadro 6 se resume esta situación.

Cuadro $\mathrm{N}^{\circ} 6$. Resumen de los revenimientos presentados durante la preparación del concreto en las 30 construcciones investigadas.

Área Metropolitana de San José.

\begin{tabular}{|c|c|c|c|}
\hline $\begin{array}{c}\text { Revenimiento } \\
(\mathrm{cm})\end{array}$ & $\begin{array}{c}\text { Cantidad } \\
\text { de obras }\end{array}$ & $\%$ de obras & Conclusión \\
\hline menor a 7,5 & 4 & 13 & mezcla muy seca \\
\hline $7.5-15,0$ & 5 & 17 & mezcla adecuada \\
\hline mayor de 15,0 & 21 & 70 & mezcla muy húmeda \\
\hline
\end{tabular}

Larelación entre las resistencias a la compresión a 28 dias y los revenimientos correspondientes se muestræn el gráfico 1 . 
Gráfico 1. Revenimiento vrs. Resistencia promedio a 28 dias para las 30 construcciones muestreadas.

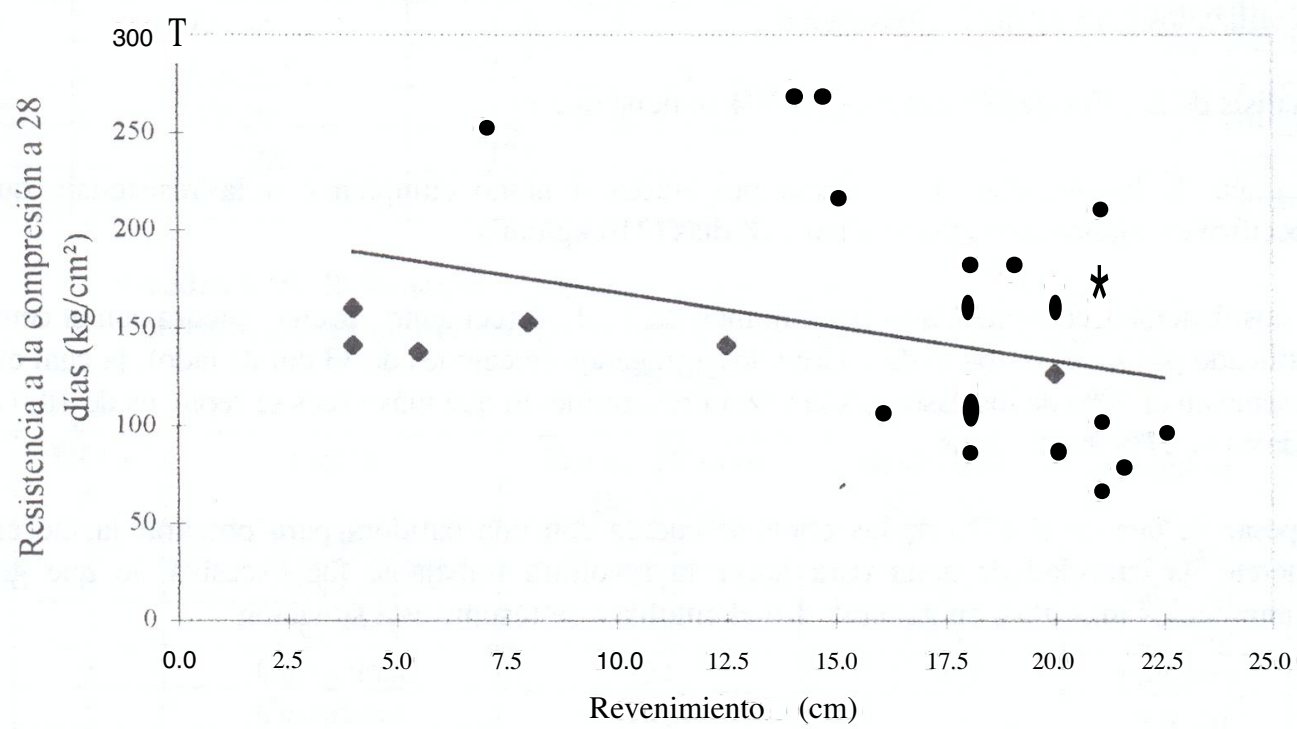

En lo que respecta al mortero. del análisis del cuadro $\mathrm{N}^{\circ} 5$ se tiene que:

- De las 20 construcciones que cuentan con una batidora para hacer concreto, sólo el $35 \%$ la utiliz2 para fabricar mortero. el $65 \%$ restante lo hace en forma manual.

- La dosificación volumétrica más común es de 1:3 (cemento: arena). la cual se presenta en el $53 \%$ de las construcciones. En ninguno de los casos se utiliza cal.

- Del total de las construcciones, el $57 \%$ de ellas fabricó la mezcla de mortero de pega en forma manual.

En los cuadros $\mathrm{N}^{\circ} 7$ y $\mathrm{N}^{\circ} 8$ se presentall los datos obtenidos en el laboratorio que caracterizan a cada uno de los agregados (fino y grueso) de las 30 construcciones.

Cuadro $N^{\circ} 7$. Resumen de las características más importantes relacionadas con el agregado fino encontrado en las 30 viviendas. Área Metropolitana de San José.

\begin{tabular}{|c|c|c|c|c|c}
\hline Obra\# & Fuente & $\sim \mathrm{s}$ & $\% \mathrm{D}$ & M.F. & Contaminación \\
\hline 1 & Taio Ochomogo & 2.0 & 13.0 & 3.2 & $<500$ p.p.m. \\
\hline 2 & Taio Ochomogo & 2,0 & 13,2 & 3.1 & $<500$ p.p.m. \\
\hline 3 & Taio Comagsa & 1.9 & 15.3 & 3,3 & $<500$ p.p.m. \\
\hline 4 & Taio Electriona & 20 & 12.6 & 30 & $<500$ p.p.m. \\
\hline 5 & Taio Cerro Minas & 1.9 & 14,1 & 3,5 & $<500$ P.P.m. \\
\hline 6 & Taio Ochomogo & 20 & 13.5 & 3,2 & $<500$ p.p.m. \\
\hline 7 & Taio Cerro Minas & 20 & 13.1 & 3,1 & $<500$ p.p.m. \\
\hline 8 & Taio Cerro Minas & 2.0 & 14.9 & 3.2 & $<500$ p.p.m. \\
\hline
\end{tabular}


Gráfico 1. Revenimiento vrs. Resistencia promedio a 28 dias para las 30 construcciones muestreadas.

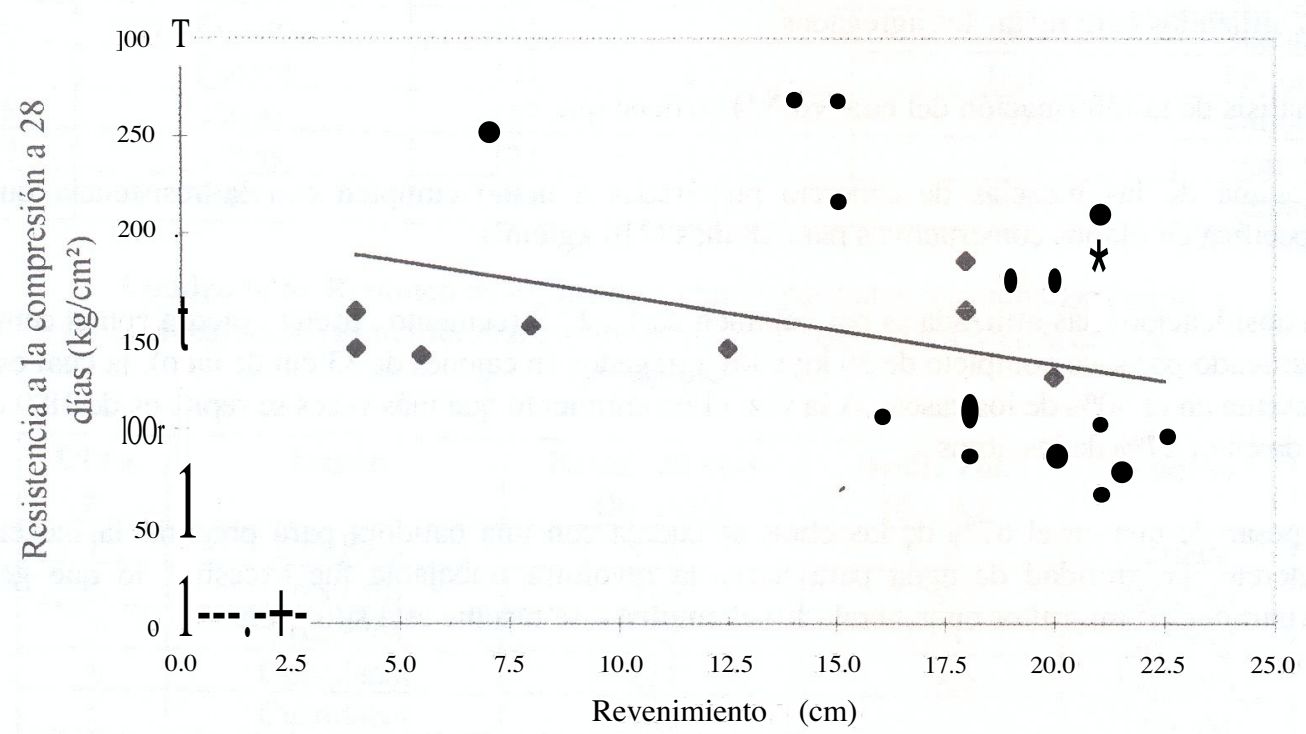

En lo que respecta al mortero. del análisis del cuadro $\mathrm{N}^{\circ} \mathrm{S}$ se tiene que:

- De las 20 construcciones que cuentan con una batidora para hacer concreto, sólo el 35\% la utiliza para fabricar mortero. el $65 \%$ restante lo hace en forma manual.

- La dosificación volumétrica más común es de 1:3 (cemento: arena). la cual se presenta en el 53\% de las construcciones. En ninguno de los casos se utiliza cal.

- Del total de las construcciones, el $57 \%$ de ellas fabricó la mezcla de mortero de pega en fonna manual.

En los cuadros $\mathrm{N}^{\circ} 7$ y $\mathrm{N}^{\circ} 8$ se presentan los datos obtenidos en el laboratorio que caracterizan a cada uno de los agregados (fino y grueso) de las 30 construcciones.

Cuadro Nº7. Resumen de las características más importantes relacionadas con el agregado fino encontrado en las 30 viviendas. Área Metropolitana de San José.

\begin{tabular}{|c|c|c|c|c|c|}
\hline Obra \# & Fuente & $\sim \mathrm{S}$ & $\% \mathrm{D}$ & M.F. & Contaminación \\
\hline 1 & Taio Ochomogo & 2.0 & 13.0 & 3.2 & $<500$ O.O.m. \\
\hline 2 & Tajo Ochomogo & 2.0 & 13.2 & 3,1 & $<500$ D.D.m. \\
\hline 3 & Taio Comagsa & 1.9 & 15.3 & 3.3 & $<500$ O.O.m. \\
\hline 4 & Tajo Electriona & 20 & 12,6 & 30 & $<500$ D.D.m. \\
\hline 5 & Taio Cerro Minas & 1,9 & 14,1 & 3,5 & < 500 O.O.m. \\
\hline 6 & Tajo Ochomogo & 20 & 13.5 & 32 & $<500$ D.D.m. \\
\hline 7 & Taio Cerro Minas & 20 & 13.1 & 3,1 & < 500 o.o.m. \\
\hline 8 & Taio Cerro Minas & 20 & 14.9 & 3.2 & < 500 D.D.m. \\
\hline
\end{tabular}




\begin{tabular}{|c|c|c|c|c|c|}
\hline 9 & Tajo Cerro Minas & 2,1 & 12,4 & 3,0 & $<500$ p.p.m. \\
\hline 10 & Tajo Electriona & 2,1 & 11.8 & 2.8 & $<500$ p.p.m. \\
\hline 11 & Tajo Cerro Minas & 2,0 & 156 & 2,9 & $<500$ p.p.m. \\
\hline 12 & Tajo Ochomogo & 20 & 13.6 & 32 & $<500$ p.p.m. \\
\hline 13 & Tajo Cerro Minas & 2,0 & 14,0 & 2,9 & $<500$ p.p.m. \\
\hline 14 & Tajo Cerro Minas & 20 & 13.7 & 3.7 & $<500$ p.p.m. \\
\hline 15 & Tajo Electriolla & 20 & 13.4 & 3,0 & $<500$ p.p.111. \\
\hline 16 & Tajo Cerro Minas & 2,0 & 14,0 & 3,3 & $<500$ p.p.m. \\
\hline 17 & Tajo Cerro Minas & 1,9 & 162 & 29 & $<500$ p.p.m. \\
\hline 18 & Tajo Electriona & 2,1 & 12,0 & 30 & $<500$ p.p.m. \\
\hline 19 & Río Ochomogo & 23 & 6,1 & 32 & $>500$ p.p.m. \\
\hline 20 & Tajo Pedregal & 1,9 & 152 & 3,3 & $<500$ p.p.m. \\
\hline 21 & Tajo Cerro Minas & 2.0 & 13,8 & 32 & $<500$ p.p.m. \\
\hline 22 & Tajo Pedregal & 20 & 14,1 & 35 & $<500$ o.p.m. \\
\hline 23 & Tajo La Garita & 2.2 & 9,2 & 2,5 & $<500$ p.p.m. \\
\hline 24 & Tajo Cerro Minas & 2,1 & 10,8 & 26 & $<500$ p.p.m. \\
\hline 25 & Tajo El Común & 2,2 & 93 & 30 & $<500$ p.p.m. \\
\hline 26 & Tajo La Garita & 2,1 & 13.1 & 29 & $<500$ p.p.m. \\
\hline 27 & Tajo El Común & 22 & 10,1 & 3,0 & $<500$ p.p.m. \\
\hline 28 & Tajo La Garita & 2,0 & 14,1 & 27 & $<500$ p.p.m. \\
\hline 29 & Tajo Comagsa & 2,0 & 134 & 3,1 & $<500$ p.p.m. \\
\hline 30 & Tajo Comagsa & 2,0 & 13,8 & 2,9 & $<500$ p.p.m. \\
\hline
\end{tabular}

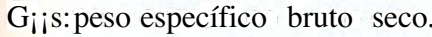

$\%$ D: absorción.
M.F.: módulo de finura.

p.p.m.: partículas por millón.

Cuadro $\mathrm{N}^{\circ} 8$. Resumen de las características más importantes relacionadas con el agregado grueso encontrado en las 30 viviendas. Área Metropolitana de San José.

\begin{tabular}{|c|c|c|c|c|c|}
\hline Obra \# & Fuente & $\sim \mathrm{s}$ & $\% \mathrm{D}$ & $\begin{array}{c}\% \text { Abr. } \\
100 \text { rey. }\end{array}$ & $\begin{array}{c}\% \text { Abr. } \\
500 \text { rey. }\end{array}$ \\
\hline 1 & Tajo Ochomogo & 24 & 67 & 5 & 20 \\
\hline 2 & Tajo Ochomogo & 24 & 44 & 5 & 22 \\
\hline 3 & Tajo Comagsa & 24 & 36 & 4 & 17 \\
\hline 4 & Tajo Electriolla & 24 & 42 & 5 & 21 \\
\hline 5 & TalO Cerro Minas & 24 & 45 & 3 & 16 \\
\hline 6 & Tajo Ochomogo & 24 & 56 & 6 & 19 \\
\hline 7 & Tajo Cerro Minas & 24 & 67 & 5 & 20 \\
\hline 8 & Tajo Cerro Minas & 23 & 7.0 & 5 & 16 \\
\hline 9 & Tajo Cerro Minas & 25 & 36 & 5 & 21 \\
\hline 10 & Tajo Electriona & 25 & 41 & 4 & 18 \\
\hline 11 & Tajo Cerro Minas & 25 & 36 & 7 & 17 \\
\hline 12 & Tajo Ochomogo & 25 & 50 & 6 & 16 \\
\hline 13 & Tajo Cerro Minas & 24 & 8,1 & 4 & 21 \\
\hline 14 & Tajo Cerro Minas & 25 & 38 & 4 & 18 \\
\hline
\end{tabular}




\begin{tabular}{|l|c|c|c|c|c|}
\hline 15 & Tajo Electriona & 2.4 & 4,0 & 7 & 18 \\
\hline 16 & Tajo Cerro Minas & 2.6 & 3.4 & 4 & 19 \\
\hline 17 & Tajo Cerro Minas & 2,4 & 5,6 & 6 & 16 \\
\hline 18 & Tajo Electriona & 2,3 & 3.8 & 7 & 20 \\
\hline 19 & Tajo La Pista & 2,4 & 4.7 & 7 & 27 \\
\hline 20 & Tajo Pedregal & 2.3 & 5,7 & 4 & 22 \\
\hline 21 & Tajo Cerro Minas & 2.5 & 4.1 & 5 & 15 \\
\hline 22 & Tajo Pedregal & 23 & 6.1 & 5 & 22 \\
\hline 23 & Tajo La Garita & 2.1 & 8,9 & 7 & 29 \\
\hline 24 & Tajo Cerro Minas & 2.4 & 6.7 & 9 & 17 \\
\hline 25 & Tajo El Común & 2.3 & 5,8 & 6 & 21 \\
\hline 26 & Tajo La Garita & 2.3 & 6.3 & 6 & 24 \\
\hline 27 & Tajo El ComÚn & 2,3 & 8,1 & 8 & 18 \\
\hline 28 & Tajo La Garita & 2,2 & 5,4 & 4 & 22 \\
\hline 29 & Tajo Comagsa & 2.4 & 6.7 & 3 & 16 \\
\hline 30 & Tajo Comagsa & 2.4 & 6.8 & 6 & 19 \\
\hline
\end{tabular}

$\sim \mathrm{s}$ : peso espeCÍfico bruto seco.

\%D: absorción.

Los módulos de finura (M.F.) del cuadro 7 se obtuvieron a partir de pruebas de laboratorio con material seco y sin lavar.

Del cuadro $\mathrm{N}^{\circ} 7$ se destaca lo siguiente:

- Solamente en una construcción (la \# 19), se utilizó arena de río, y es el único agregado fino que presenta una contaminación apreciable.

- El GBs más alto es de 2,3 y el más bajo igual a 1,9. En cada caso la absorción alcanza $6.1 \%$ y $16,2 \%$ respectivamente, valores que representan el \% m más bajo y el más alto.

Para el caso de la piedra, en el cuadro $\mathrm{N}^{\circ} 8$ sobresalen los aspectos que a continuación se exponen:

- El $G_{B S}$ más alto fue de 2,6 y el más bajo igual a 2.1. Sus correspondientes valores de absorción fueron $3,4 \%$ y $8.9 \%$.

- En la prueba de abrasión con la máquina Los Ángeles se obtienen dos propiedades del agregado conocidas como tenacidad a 100 revoluciones y abrasión a 500 revoluciones. Para la primera $\mathrm{cl}$ porcentaje de pérdida debe ser menor al $10 \%$ y en el segundo caso menor al $40 \%$ para pavimentos. o al $50 \%$ para concreto hidráulico estructural. Se observa que todos los agregados gruesos analizados cumplen con estas dos propiedades. Sin embargo, esto no indica que realmente el agregado sea de buena calidad. debido a que las especificaciones que rigen esta prueba son muy conservadoras.

\section{ANALISIS ESTADISTICO DE LOS RESULTADOS PARA EL CONCRETO $y$ EL MORTERO}

El análisis estadistico de la muestra constituida por 30 viviendas escogidas al azar dentro del Área Metropolitana de San José. refleja que el concreto elaborado en obra. como promedio, es de baja calidad. Del cuadro $\mathrm{N}^{\circ} 9$ se obtiene que el promedio de la muestra sólo llega a alcanzar el 69\% de la resistencia especificada para 28 dias. Además, si a los $146 \mathrm{kglcm}^{2}$ se le adiciona el 
valor de la desviación estándar de $55 \mathrm{~kg} / \mathrm{cm}^{2}$, tampocose cumplirá con $\mathrm{f}^{\prime} \mathrm{c}=21 \mathrm{O} \mathrm{kg} / \mathrm{cm}^{2}$.

Porotro lado, en la desviación estándar, en el coeficiente de variación y en la amplitud se refleja la gran variabilidad de los resultados, lo que indica que los procesos de fabricación del concreto en el sitio son muy irregulares.

Cuadro $\mathrm{N}^{\circ}$ 9. Resultados del análisis estadistico del concreto estructural correspondiente a 30 viviendas ubicadas en el Área Metropolitana de San José.

\begin{tabular}{|c|c|}
\hline Medida estadística & Valor calculado \\
\hline Promedio & $146 \mathrm{~kg} / \mathrm{cm}^{2}$ \\
Desviación estándar & $55 \mathrm{~kg} / \mathrm{cm}^{2}$ \\
Coeficiente de variación & $37,9 \%$ \\
Mediana & $140 \mathrm{~kg} / \mathrm{cm}^{2}$ \\
Máximo & $272 \mathrm{~kg} / \mathrm{cm}^{2}$ \\
Mínimo & $67 \mathrm{~kg} / \mathrm{cm}^{2}$ \\
Amplitud & $205 \mathrm{~kg} / \mathrm{cm}^{2}$ \\
\hline
\end{tabular}

Cuadro 10. Resultados del análisis estadistico del mortero de pega correspondiente a 30 viviendas ubicadas en el Área Metropolitana de San José.

\begin{tabular}{|c|c|}
\hline Medida estadística & Valor calculado \\
\hline Promedio & $186 \mathrm{~kg} / \mathrm{cm}^{2}$ \\
Desviación estándar & $49 \mathrm{~kg} / \mathrm{cm}^{2}$ \\
Coeficiente de variación & $26,2 \%$ \\
Mediana & $179 \mathrm{~kg} / \mathrm{cm}^{2}$ \\
Máximo & $306 \mathrm{~kg} / \mathrm{cm}^{2}$ \\
Mínimo & $114 \mathrm{~kg} / \mathrm{cm}^{2}$ \\
Amplitud & $192 \mathrm{~kg} / \mathrm{cm}^{2}$ \\
\hline
\end{tabular}

En el mortero, el promedio sí cumple con las especificaciones (para tipo $M Y S$ ) pero, aunque la variabilidad que se reflt .ia en las distintas medidas estadisticas es menor que en el concreto, su magnitud sigue siendo muy alta.

Algunos factores presentes en la totalidad de las construcciones, y que no benefician de ninguna manera la resistencia del concreto o delmortero son:
- No hubo control del agua; no se utilizó medida alguna para dosificar el agua con respecto al peso del cemento y tampoco se tomó en cuenta el agua presente en los agregados.

- En ninguno de los casos existe memoria de cálculo que respalde el diseño de mezcla.

- No se almacenó el cemento en obra en fonna adecuada. Quizás esto obedece a que el periodo de rotación es muy rápido (inferior a 15 dias). 
- En todos los casos se desconoce la granulometría que presentan los agregados.

- En ninguna de las obras se utilizó algún tipo de vibrador para consolidar y colocar el concreto.

\section{CONCLUSIONES}

Quizás el mayor problema con los agregados es que no existe en obra control alguno sobre su calidad. Generalmente, el profesional a cargo no se encuentra en la constmcción a la hora de recibir el material y la persona que lo $h \sim \backslash \sim \sim n$ Qrm(llm nt-no esttí cll ncitil\&l, ni se preocupa por exigir la calidad del agregado que se solicitó.

De acuerdo con los datos obtenidos, se evidencia la relación directa que existe entre el peso del espécimen y su resistencia a la compresión, siendo más marcada para el caso de los cilindros de concreto. ya que estos al contener agregado grueso poseen una resistencia que es ftillción de la calidad de esa piedra. Además entre más pesado es un agregado, siempre y cuando no sea reactivo, es mejor su calidad, debido a que esto es reflejo de tilla menor porosidad.

Con base en el estudio estadístico, la probabilidad de obtener (en el Área Metropolitana) una mezcla de concreto que cunlpla con la resistencia especificada a 28 dias es del $11,4 \%$. De igual forma, la probabilidad de lograr un mortero tipo $\mathrm{M}$ es del $58,7 \%$. Yuno del tipo $\mathrm{S}$ es del $88,9 \%$.

En esta investigación se encontró que el $70 \%$ de las obras presentaron revenimientos superiores a los $15 \mathrm{~cm}$. Se verificó que las mezclas presentaban problemas de granulometría y que estaban pasadas de agua. En cada una de las 30 constmcciones no se tuvo el mínimo control de la relación agua/cemento.
El mayor revenimiento que se dio fue de 22,5 $\mathrm{cm}$ y el menor de $4,0 \mathrm{~cm}$, lo que en ambos casos no es recomendable, ya que indican mezclas muy húmedas o muy secas.

El concreto más resistente $\left(272 \mathrm{kglcm}^{2}\right)$ telúa una dosificación volumétrica de $1: 1.5: 3$ (cemento: arena: piedra), un reveninúento de $14,0 \mathrm{~cm}$ y fue preparado en batidora. Contrariamente el concreto más deficiente (67 $\mathrm{kglcm}^{2}$ ) presentaba una dosificación volumétrica de $1: 3: 3$ (e: A : P), un revenimiento de $21,0 \mathrm{~cm}$ y fue hecho en forma manual. Estos dos ejemplos son evidencia

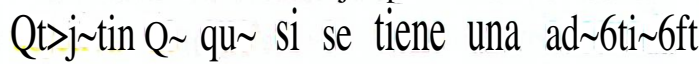
dosificación y un revenimiento aceptable, se pueden esperar buenos resultados.

El mortero más resistente $\left(306 \mathrm{kglcm}^{2}\right)$ tenia una dosificación volumétrica de $1: 2$ (cemento: arena) y fue preparado en fonna manual. El más deficiente $\left(114 \mathrm{~kg} / \mathrm{cm}^{2}\right)$ presentó una dosificación volumétrica de 1 : 3,5 (C : A) y fue hecho también manualmente.

Para el cemento portland tipo 1 MP los resultados de las pruebas de laboratorio fueron los siguientes:

- AS.T.M. C 187: la consistencia nonnal se obtuvo para un $28,5 \%$ de agua en peso con relación al cemento.

- AS.T.M. C 191: el tiempo de fraguado inicial se dio para un tiempo de 144,6 minutos.

- AS.T.M. C 188: el peso específico presentó un valor de 2,96.

Los valores de resistencia obtenidos corresponden a mezclas de mortero y concreto que fueron curados durante 28 dias. $\mathrm{Si}$ se toma en cuenta que el curado en obra en este tipo de construcciones es alrededor de 7 dias, es de esperar que las resistencias alcanzadas en obra estén por debajo de los valores 
encontrados en el laboratorio al fallar los testigosde prueba.

\section{RECOMENDACIONES}

Cuandose dosifique por volumen. no se deben mezclar distintos tipos de medidas (cajones, baldes,carretillos) pues al hacerlo se modifica la dosificación a cumplir.

En cada obra se deben tener recipientes para dosificarel agua. Estos deben tener unidades de litros para medir volúmenes, con el fin de obtener directamente el peso del agua en kilogramos.

El ingeniero o arquitecto no debe limitarse a labores administrativas, ya que en la construcción es donde se presentan las verdaderas dificultades y donde se necesita que una persona capacitada pueda dar solución a los distintos problemas que se presentan. Ante la ley él es el responsable de la calidad de la obra.

Es necesario que se imponga como requisito en todo tipo de construcción, el control de calidad, como mínimo, para el concreto y el mortero. Con los resultados de las pruebas de laboratorio, el profesional a cargo puede enterarse de la calidad resultante y si está cumpliendo con las especificaciones, o si existe la necesidad de hacer variaciones con el finde obtener un producto seguro y resistente.

Se debe realizar la prueba de revenllluento cada vez que se preparan batidas nuevas de mezcla de concreto en el sitio. El equipo necesario es sencillo de utilizar, lo que facilita el entrenamiento de los operarios y maestros de obra para que puedan realizarla.
Cuando el cemento a utilizar sea portland tipo $1 \mathrm{\Lambda P}$, las dosificaciones por volumen usuales para portland tipo 1 (concreto y mortero) pueden seguir siendo utilizadas. siempre y cuando se especifique que la proporción correspondiente al cemento se debe interpretar como sacos enteros de $50 \mathrm{~kg}$ y la dosificación de los agregados en cajones de $0.0333 \mathrm{~m}^{3}$ de volumen, o sea recipientes cúbicos de aproximadamente $33 \mathrm{~cm}$ de lado interno. Esta fonna de dosificar es muy práctica y de fácil entendimiento para los trabajadores en el sitio.

\section{BIBLIOGRAFÍA}

1. Anlerican Society for Testing and Materials. Specifications, test methods, practices, classifications and definitions. U. S.A, 1990.

2. Araya R. M. Control de calidad del concreto estructural y del mortero de pega en viviendas. Tesis Lic. lng. Civil. Universidad de Costa Rica, 1998.

3. Asociación AC.I., C. R. CÓmo hacer buenas mezclas de concreto. lera edición. San José, Costa Rica. 1992.

4. Colegio Federado de Ingenieros y Arquitectos de Costa Rica. Código Sísmico de Costa Rica. Editorial Tecnológica de Costa Rica, 1987.

5. Comité A.c.I. 704. Control de calidad del concreto. Instituto Mexicano del Cemento y el Concreto. Editorial Limusa. México. 1990.

6. Diario oficial La Gaceta,\# 167. San José, Costa Rica, 5 de setiembre de 1990.

7. Gronemeyer K.. K. Estudio de prefactibilidad para la aplicación del concreto premezclado en V'iviendas de una 
$y$ dos plantas. Tesis Lic. Ing. Civil, Universidad de Costa Rica. 1995.

8. Mendenhall. W. introducción a la probabilidad i: la estadística. Grupo Editorial lberoamérica. México D. F., México. 1987.

9. Merrit S.. F. Enciclopedia de la construcción, arquitectura e ingeniería. Editorial Océano. Barcelona, España, 1991.

10. Muñoz U.. F. M. Apuntes de clase del curso Afateriales de Construcción. Escuela de Ingeniería Civil, Universidad de Costa Rica. 1995.

11. Muñoz U.. F. M. iVezclas de concreto con detergentes como aditivos. 1. N. 1. 1., Universidad de Costa Rica. 1994.

12. Muñoz V.. J. influencia de la cal en la resistencia a compresión de morteros.
Tesis Lic. Ing. Civil, Universidad de Costa Rica. 1995.

13. Neville A., M. Tecnología del concreto. Instituto Mexicano del Cemento y el Concreto, Editorial Limusa, México, 1988.

14. Payá P., M. Hormigón vibrado y hormigones especiales. Ediciones CEAC, Barcelona. España, 1975.

15. Pereira S., 1. Control de calidad en viviendas de uno y dos pisos. Tesis Lic. Ing. Civil, Universidad de Costa Rica, 1994,.

16. Productos de Concreto. Catálogo general. $14^{\mathrm{a}}$ edición. San José. Costa Rica, 1992.

17. Rojas R., A. Evaluación del comportamiento del mortero de albañilería, con adherente y larga vida. Tesis Lic. Ing. Civil. Uruversidad de Costa Rica, 1993. 